\title{
STUDY ON ECOLOGICAL RISK ASSESSMENT OF DIFFERENT LAND USE TYPES BASED ON HMER MODEL - TAKING THE DAXIA RIVER IN GANSU, CHINA AS AN EXAMPLE
}

\author{
WANG, S. - ZHANG, C. ${ }^{*}-$ JI, H. - ZHANG, Y. - LOU, T. \\ College of Computer Science \& Engineering, Northwest Normal University, Lanzhou 730070, \\ China \\ *Corresponding author \\ e-mail: dearzhangchang@qq.com \\ (Received 10 $0^{\text {th }}$ Jun 2019; accepted 28 ${ }^{\text {th }}$ Aug 2019)
}

\begin{abstract}
The effective heavy metal content of soil indicates ecological environmental risks. The study takes the typical watershed area in northwestern China as a research area. The real-time and rapid risk assessment of ecological environment can be achieved through the monitoring value of normalized difference vegetation index (NDVI) of remote sensing information and environmental factors, and a heavy metal ecological risk assessment model (HMER) for the evaluation of the watershed is built. The research results show that the change of the effective heavy metal content in different areas of the watershed is affected to some extent by the influence of geographical location and human activities. Among them NDVI and the content of heavy metals, precipitation and temperature, and a nonlinear relationship between the average heavy metal content and the ecological risk index HRI. The establishment of HMER research model is applied to different land use types to evaluate the ecological risk level of the watershed, explore the ecological risk level of different land use types in the same region, and in different regions. The results of the study emphasize that the risk assessment of the watershed can be carried out through remote sensing information and environmental factors.
\end{abstract}

Keywords: soil, heavy metals, HMER, land use type, NDVI

\section{Introduction}

Soil safety is closely related to agricultural production, food safety, human health and ecological health (Shi et al., 2019). At present, about 20\% of China's land ecological environment is under high risk, with a total area of about 0.11 billion $\mathrm{km}^{2}$ (Liu and Zhang, 2010). Among them, the effective pollution status of heavy metals in soil environment has received increasing attention (Yang et al., 2018). Studies have shown that heavy metals pose a high risk of environmental exposure by spreading and accumulating between plants (the main pathways includes crops and vegetables) and animals (Sawut et al., 2018). The heavy metal environmental hazard released by the ecological chain link process has a negative impact on the viability of urban trees (Yu et al., 2018). Frequent human activities accelerate the release of heavy metal activity, causing heavy metals in the soil to pose a public health threat (Doabi et al., 2018).

In different land use types, soil moisture content will change (Tang et al., 2018). Physical properties such as soil texture, porosity, structure, and chemical properties such as absorption and cushioning are also different (Liang et al., 2018). As the content of heavy metals in soil is related to these properties of soil, there are differences in the distribution and quantity accumulation of heavy metals in soil of different land use types (Su et al., 2013), which leads to the phenomenon that the content of heavy metals in soil of different land types is different (Wang et al., 2016). Some scholars have carried out ecological assessment studies on the production of heavy metals in different 
land use types in the same area (Zhou et al., 2017). Some scholars have used different evaluation methods to study the status of heavy metal pollution (Liu et al., 2013). The reasons for these differences and the relationship between soil physical and chemical properties and heavy metals under different land use types need further verification ( $\mathrm{Wu}$ et al., 2018). Traditional evaluation methods all require field sampling, but due to the weather, geographical location and other factors, the difficulty of sampling is increased, and the sample processing time is also relatively long. These factors cause great obstacles to further study and understanding of regional heavy metal ecological risk.

This paper studies the impact of human activities on environmental and ecological risks from the perspective of land use types and the correlation between climatic and environmental factors and heavy metals. The precipitation, temperature and NDVI of the study area are analyzed. The extraction and comparison of different land use types were carried out, and then the remote sensing data and environmental data and the ecological assessment model of the average content of heavy metals were established in the soil over a long period of time. The relationship between different land use types and ecological risk index was explored, and then the remote sensing data was used to estimate the distribution of heavy metals in the basin and construct an ecological risk assessment model. The research methods are applied to different land use types in the same area and the same land use types in different areas to determine their ecological risk levels. Because of the difficulty of sampling different land use types in the basin area, it is more accurate to carry out ecological risk assessment for the study basin area. The research results provide a theoretical reference for the assessment of soil heavy metal ecological risk in small and medium watersheds, the prevention and treatment of heavy metals in farmland and the methods of restoration.

\section{Materials and methods}

\section{Research area overview}

The research area is the Daxia River which flows through the southwestern part of Gansu Province in China. The study area is located between $102^{\circ} 02^{\prime} \sim 103^{\circ} 23^{\prime}$ East longitudes and $34^{\circ} 51^{\prime} \sim 35^{\circ} 48^{\prime}$ 'North latitude. There are two river sources in the Daxia River, namely Xiahe and Luohe (Yang, 2013). The Daxia River originates in the southern part of Tongren County and the west of Xiahe County in Qinghai Province, and flows through Qingshui Gorge in Xiahe County, passes through Tumen Gate and enters Maji Town of Linxia County, and passes through Yinji and Xinji Towns into Linxia City. From the estuary, the river enters the Liujiaxia Reservoir and merges into the Yellow River. It is called "the first-level tributary of the upper reaches of the Yellow River" (Wang, 2016). According to the water conservancy department in Linxia Prefecture, $45.5 \%$ of the river water resources in the area are affected by different levels of anthropogenic pollution, and $11.6 \%$ has water quality that is lower than the drinking standard. More than $90 \%$ of municipal water resources are threatened by water security (Chen, 2015). In terms of the geographical distribution of water pollution, the water quality of the main stream is higher than in the tributaries, and the upstream water quality is better than the downstream. A major source of water pollution is the mines (Luo, 2017). Water conservation in the Daxia River Basin is regarded as an important foundation for the sustainable social and economic development in the ethnic minority areas (Lin, 2015). 


\section{Source and processing of remote sensing data}

Remote-sensing data are mainly provided by the Geospatial Data Cloud (http://www.gscloud.cn/) and the Landsat 8 OLI_Thermal Infrared Sensor (TIRS) remote sensing satellite data from the LANDSAT series of data provided by the United States Geological Survey (USGS) (https://earthexplorer.usgs.gov/). The applied remote sensing image is in the period from 2015 (January to December) to 2017 (January to December), and its spatial resolution is $30 \mathrm{~m} \times 30 \mathrm{~m}$. The ENVI software was used to calculate the corresponding NDVI values for radiometric calibration, image mosaic, image cropping, and atmospheric correction on the smoke image of the study area, and to remove the outliers and center standardization. The NDVI is defined as the ratio of the difference between near-infrared (NIR) and visible-red-red (R) bands and the sum of the two bands, NDVI = (NIR-R) / $(\mathrm{NIR}+\mathrm{R})$. In vegetation remote sensing, NDVI is the most widely used, as (1) NDVI can be applied to detect vegetation growth status, vegetation coverage, and to eliminate some radiation errors; (2) negative values indicate that the ground cover is cloud, water, snow, etc., with a high reflection for visible light; 0 means rock or bare soil, where NIR and $\mathrm{R}$ is approximately equal; positive values indicate vegetation coverage, and the positive value increases with increasing coverage (Yuan and Gu, 2018). The average monthly NDVI of the study area from 2015 (January to December) to 2017 (January to December) is shown in Figure 1.

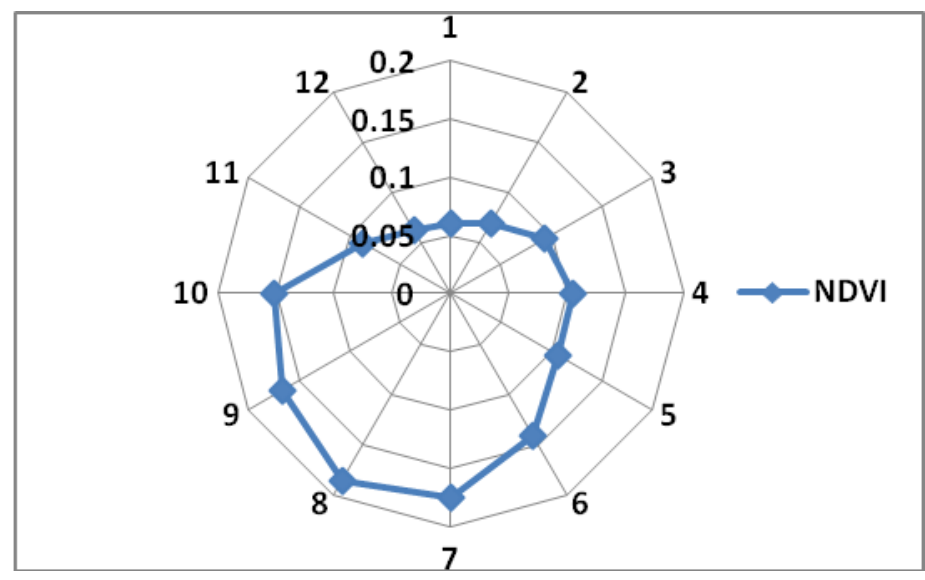

Figure 1. NDVI monthly average

\section{Source and treatment of heavy metal data}

In order to determine the number of samples more reasonably, this paper uses the Cochran method to estimate the optimal number of samples and sampling points for the construction constructed with pure random sampling of the study area. And there are 114 sampling points, including 24 fixed sampling points and 90 random sampling points. Among them, the fixed sampling point refers to the point to be collected every time, and the random sampling point is the point that is not necessarily collected every month, which can make the sample closer to the real situation. The main sampling time for this study is 2015 (January to December) to 2017 (January to December). Among them, the sample collection is performed once a month. The distribution of sampling points is shown in Figure 2. When collecting samples, a hand-held GPS device was used, and a plastic spoon was used to take $0-20 \mathrm{~cm}$ of the upper layer of the bottom 
mud. The soil sample was dried and ground, after passing through a 200-mesh nylon sieve. In addition, the sample was processed by microwave digestion. Finally, the content of heavy metals was determined by inductively coupled plasma optical emission spectrometer (ICP-OES). By combining remote sensing images and combining field visits to study areas, we learn about the main sources of heavy metals in the study area, such as plant emissions, and the use of pesticides.

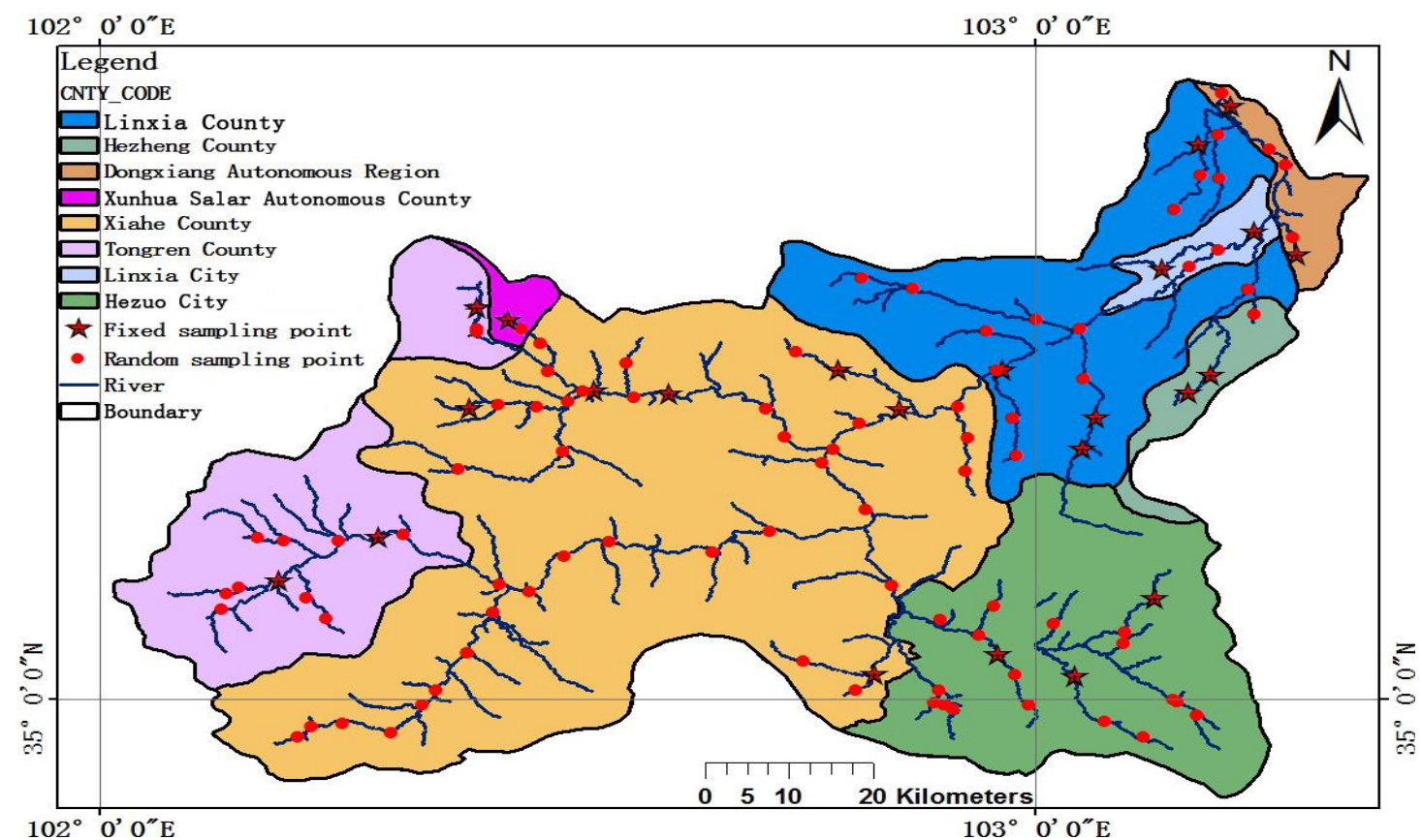

Figure 2. Study area sampling point map

\section{Source and treatment of precipitation and temperature}

Surface meteorological data from the China Meteorological Data Network (http://data.cma.cn/site/index.html) is typically used as the main source of precipitation and temperature data. Monthly average temperature and monthly average precipitation data from January 2015 to December 2017 was obtained for the study.

\section{Results and discussion}

\section{Relationship between regional factors}

\section{Impact of climatic factors on NDVI}

As the seasons change, the value of NDVI will change due to variations in moisture and temperature (Wang et al., 2018) Changes in vegetation cover are also affected by the combined or synergistic effects of hydrological conditions (such as temperature, precipitation, and humidity) (Liu et al., 2018). Due to little variation in $\mathrm{pH}$ and land use type in the study area, this paper focuses on the differences in precipitation and temperature. The amount of precipitation, temperature, and NDVI is affected to some extent by human activities. The monthly average NDVI values from January 2015 to December 2017, the monthly average precipitation and the monthly average temperature were used for correlation analysis. 
It can be observed that the correlation between monthly average NDVI value and monthly average precipitation is 0.807 , and the correlation between monthly average NDVI value and monthly mean temperature is 0.808 . The relationship between temperature and NDVI $(\mathrm{P}=0.01<0.05)$, and precipitation and NDVI $(\mathrm{P}=0.02<0.05)$ are statistically significant. Details are shown in Figure $3 a, b$.

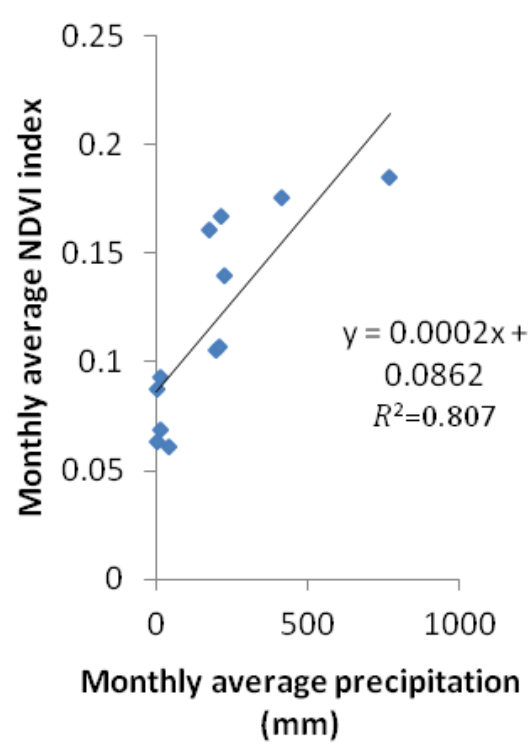

(a)

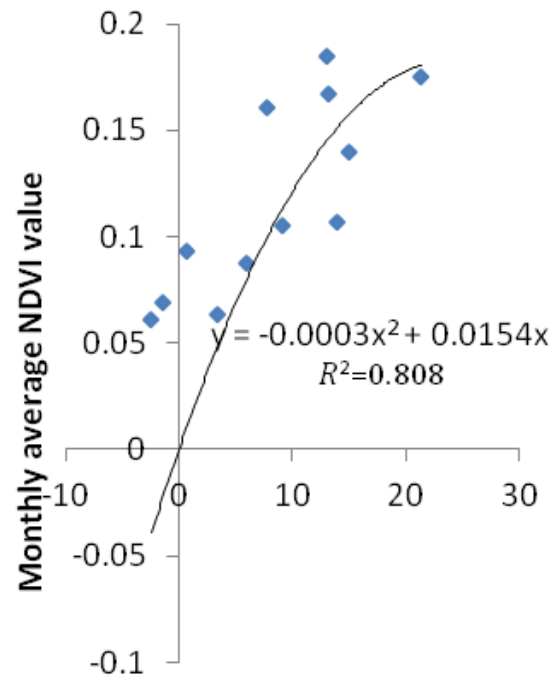

Monthly average temperature

$\left({ }^{\circ} \mathrm{C}\right)$

(b)

Figure 3. Relationship between NDVI and precipitation temperature

\section{Influence of climatic factors on heavy metal content}

Field measurements showed evidence of the presence of $\mathrm{Cd}, \mathrm{Cr}, \mathrm{Cu}, \mathrm{Pb}$, and as in the research area. The content of heavy metals is related to the migration of heavy metals. The speed of migration is linked to precipitation and temperature, and the cumulative effect of heavy metals in organisms is related to climatic factors (Ahonen et al., 2018). The soil heavy metal content is significantly correlated with the annual average precipitation and temperature at the sampling point (Zhou et al., 2018). Human activities indirectly affect the content of heavy metals by affecting climate change. The monthly average heavy metal content of the five elements was calculated, and a correlation analysis was undertaken for monthly precipitation and monthly average temperature. The correlation between monthly heavy metal content and precipitation was -0.79 , and the correlation between monthly heavy metal content and temperature was -0.784 , and the significance was sig. $=0.03$, sig. $=0.02$, respectively. The average heavy metal content in soil is negatively correlated with precipitation and temperature. Erosion and rainfall runoff will reduce the content of heavy metals (Li et al., 2011). An increase in temperature causes increased acidity and an increase in the activity of heavy metal ions, which means that the heavy metal content increases. However, the increase of heavy metal active ions means that the water solubility of heavy metal ions increases. Thus, heavy metal content can be seasonal, increasing with an increase in temperatures. The scouring effect is notable, and will reduce the heavy metal content of the soil. Details are shown in Figure $4 a, b$. 


\section{Ecological risk assessment model}

\section{Remote sensing data prediction of heavy metal average content model}

The heavy metal content, the average monthly NDVI, the monthly average precipitation, and the monthly average temperature data from 2015 (January to December) to 2017 (January to December) in the Daxia River Basin were modeled using SPSS20 statistical software. The modeling result was $R^{2}=0.866$.

$$
H=-0.002 P-54.2876 N-0.042 T+34.443
$$

where $\mathrm{H}$ is the average heavy metal content, $\mathrm{P}$ and $\mathrm{T}$ are the precipitation amount and temperature, respectively; $\mathrm{N}$ is the NDVI value.

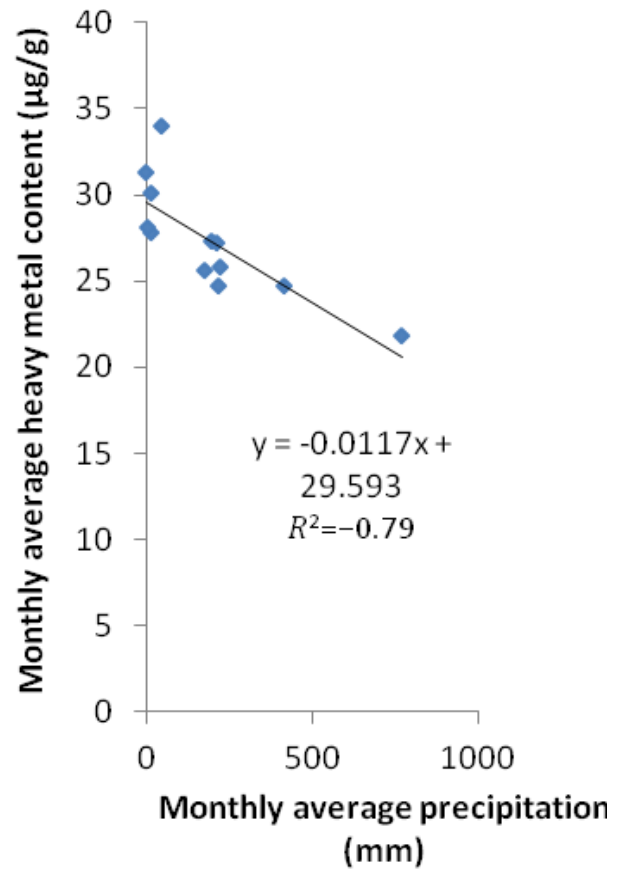

(a)

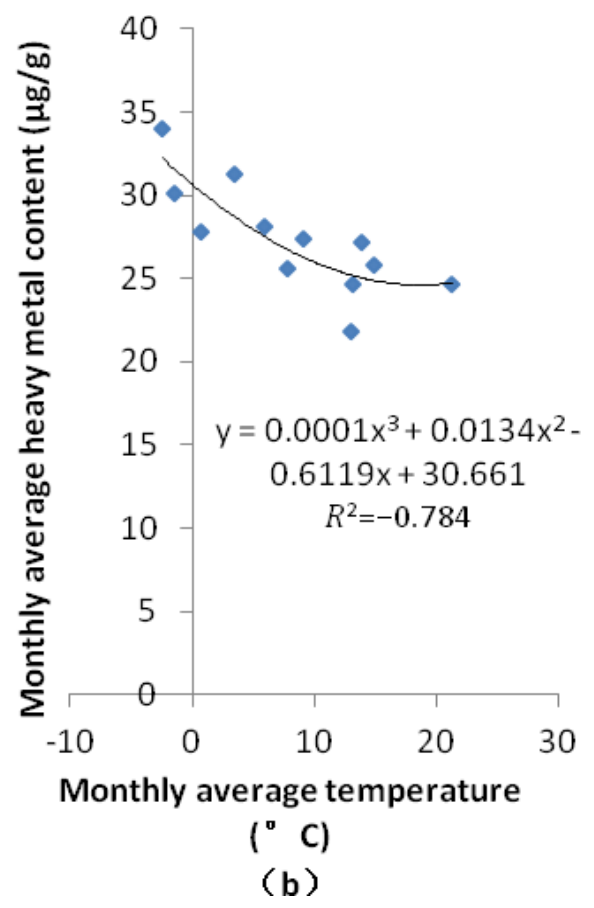

Figure 4. Relationship between monthly heavy metal content and water purification temperature

From 2015 (January to December) to 2017 (January to December), the fixed sampling points of the study area and the values of the random sampling points are measured. After treatment, the content of heavy metals was determined by inductively coupled plasma optical emission spectrometer (ICP-OES), and then the values were averaged. The comparison with the average heavy metal content of the soil predicted by Equation 1 is as follows. Equation 1 can be used to predict the average heavy metal content of the study area. From Figure 5, the average heavy metal content in August is the lowest. The vegetation in August is the lushest, and the vegetation has an inhibitory effect on the dissolution of heavy metal content in the soil. 


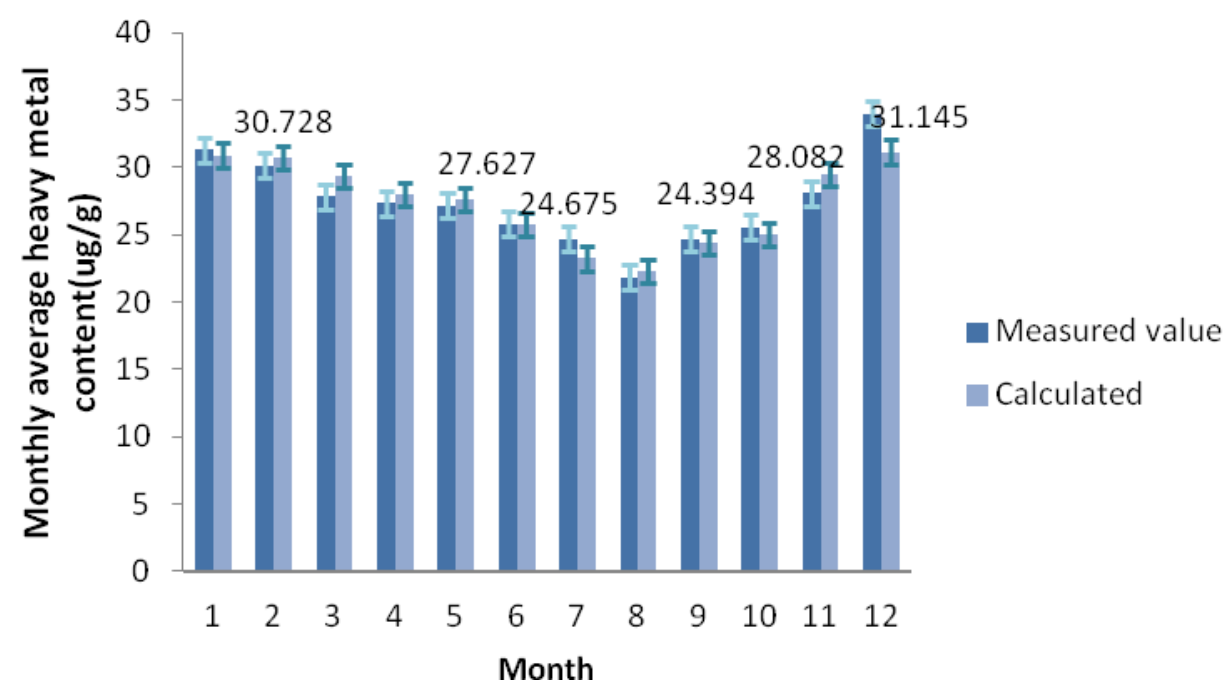

Figure 5. Comparison of measured and calculated values of monthly heavy metal content

\section{Establishment of ecological risk assessment model}

The Hakanson Ecological Risk Index is a commonly used potential ecological risk assessment index for heavy metals (Hakanson, 1980) which predicts the slow release processes and potential effects of heavy metals in the soil. In response to this potential hazard, the Swedish environmentalist Lars Hakanson introduced the potential ecological risk index (HRI) in 1980.

$$
H R I=\sum_{i=1}^{n} E_{r}^{i}=\sum_{i=1}^{n} T_{r}^{i} \cdot C_{r}^{i}=\sum_{i=1}^{n} T_{r}^{i} \cdot \frac{C_{f}^{i}}{C_{z}^{i}}
$$

where: $H R I$ is the potential ecological risk index of $\mathrm{n}$ kinds of heavy metals in the sample. $E_{r}^{i}$ is the single potential ecological risk index of the sample heavy metal $\mathrm{I} ; T_{r}^{i}$ is the toxicity response coefficient of the T heavy metal I; $C_{r}^{i}$ is the pollution coefficient of the heavy metal I; $C_{f}^{i}$ is the measured value of the heavy metal $\mathrm{i}$ in the sample, and $c_{z}^{i}$ is the specific gravity metal value of the uncontaminated sediment before industrialization. (This can also be replaced by the local sediment background value.) The geochemical background values of the heavy metals in the study area (Wang and Lian, 1993) are shown in Table 1, and the classification criteria for the potential ecological hazard index of heavy metals (Li et al., 2015) are shown in Table 2.

The size of HRI value is related to the content of heavy metal. Therefore, the average heavy metal content $\mathrm{H}$ calculated in Equation 1 is the independent variable, and the HRI calculated by Equation 2 is the dependent variable, and the linear fitting is performed. The fitting result is shown in Table 3.

Table 1. Geochemical background values of soil heavy metals in the study area (Wang and Lian, 1993)

\begin{tabular}{c|c|c|c|c|c}
\hline Project & Copper & Lead & Arsenic & Cadmium & Chromium \\
\hline Background values & 28.91 & 20.83 & 14.03 & 0.130 & 75.35 \\
\hline
\end{tabular}


Table 2. Classification criteria for potential ecological hazard index of heavy metals (Li et al., 2015)

\begin{tabular}{c|c|c|c}
\hline Risk level & $E_{r}^{i}$ & $H R I$ & Potential ecological risk \\
\hline A & $<=40$ & $<=150$ & Low \\
B & $40 \sim 80$ & $150 \sim 300$ & Medium \\
C & $80 \sim 160$ & $300 \sim 600$ & Slightly serious \\
D & $160 \sim 320$ & $600 \sim 1200$ & Serious \\
E & $>320$ & $>1200$ & Extremely serious \\
\hline
\end{tabular}

$E_{r}^{i}$ is the potential ecological hazard index of heavy metals I; HRI is the potential ecological risk index

Table 3. Fitting results of HRI and average heavy metal content

\begin{tabular}{c|c|c|c|c|c|c|c}
\hline Model type & $\begin{array}{c}\text { Linear } \\
\text { function }\end{array}$ & $\begin{array}{c}\text { Logarithmic } \\
\text { function }\end{array}$ & $\begin{array}{c}\text { Countdown } \\
\text { function }\end{array}$ & $\begin{array}{c}\text { Quadratic } \\
\text { function }\end{array}$ & $\begin{array}{c}\text { Cubic } \\
\text { function }\end{array}$ & $\begin{array}{c}\text { Composite } \\
\text { function }\end{array}$ & $\begin{array}{c}\text { Power } \\
\text { function }\end{array}$ \\
\hline Correlation & 0.731 & 0.682 & 0.629 & 0.88 & 0.893 & 0.825 & 0.785 \\
\hline
\end{tabular}

By comparing the fitting results, the cubic function fitting is ideal, the cubic formula is selected, and the specific fitting Equation 3 is the remote heavy metal ecological risk assessment model (HMER) follows:

$$
H M E R=0.02 H^{3}-0.718 H^{2}+228.034
$$

where $\mathrm{H}$ represents the average heavy metal content calculated in Equation 1; HMER represents the ecological risk assessment value, and its classification is referred to Table 2.

Using the data from 2018 (January to June) for verification, a comparison between the verification result and the sampling result in shown in Figure 6 (Numbers 1-12 and Numbers 13 to 19 are the data of the sample area and verification area, respectively). The relationship between the watershed heavy metal ecological risk index, RRI, and the meteorological remote sensing information can be expressed by Equation 3.

\section{Model application}

\section{Application of different land use type models in the same area}

Due to the relatively large difficulty of different land use types in the whole study area, this method is used to evaluate the ecological risks of different land use types.

By using ENVI software to read, inlay, trim, and atmospheric correction of remote sensing images with less cloud cover, the unsupervised classification K-Means and the combination of on-site surveys are used to classify the land as shown in Figure 7. The map is processed by Majority/Minority analysis, and the clustering process is carried out to ensure the continuity of the space. The filtering problem is also used to solve the island problem in the picture, so the image is close to the real situation. In this study, land types are mainly divided into water area, grassland, town, cultivated land and woodland, as shown in Figure 7. Among them, the NDVI value is usually in the range 
of $[-1,1]$. The negative value of the waters is normal. When the waters are extracted, the water bodies may have positive values because some vegetation grows in the water and floats on the water surface. Similarly, when extracting land use types such as grassland, woodland, cultivated land, and towns, it may be affected by the background of the plant canopy, such as soil, wet ground, snow, dead leaves, roughness, etc., and will get negative values. NDVI values of each land use type were calculated by using mask and other operations, as shown in Table 4.

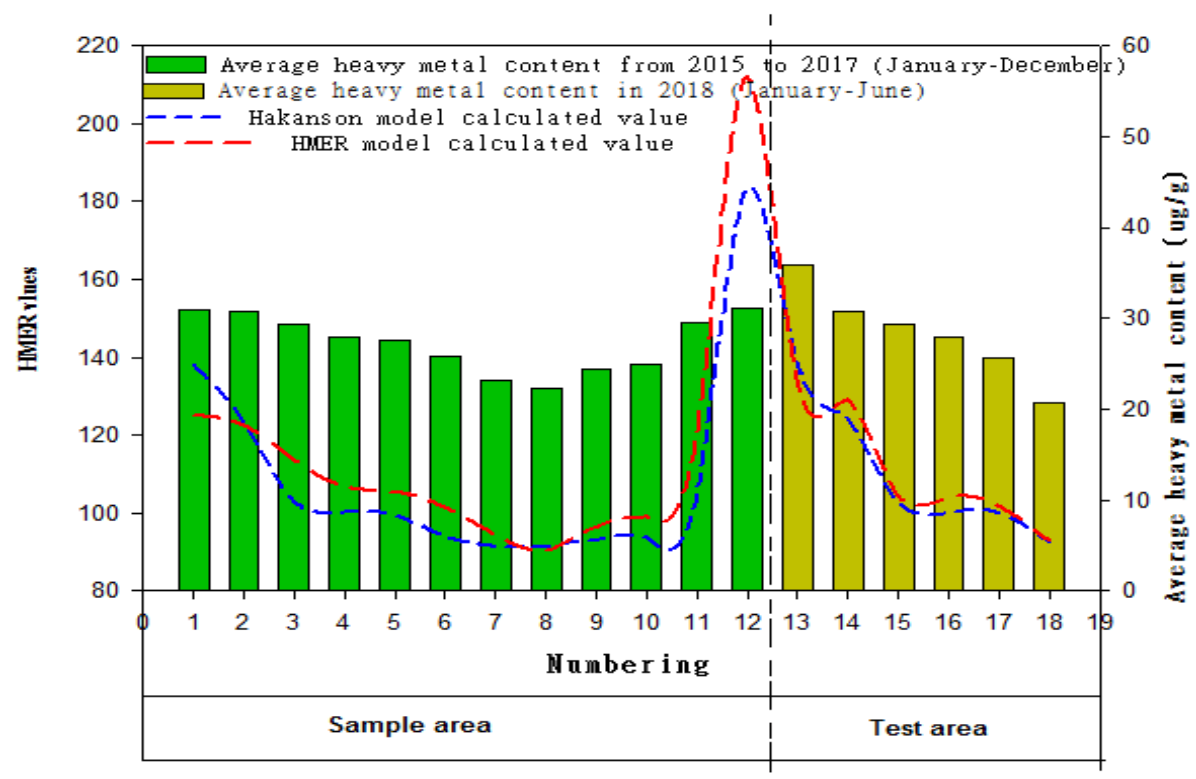

Figure 6. Comparison of the HMER model and the Hakanson model

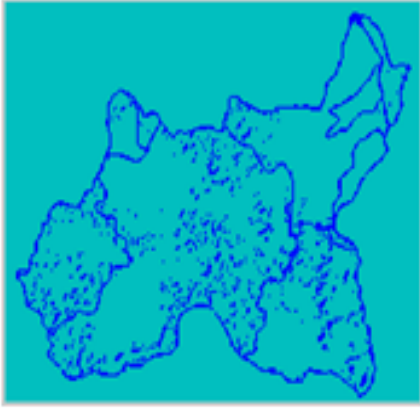

1

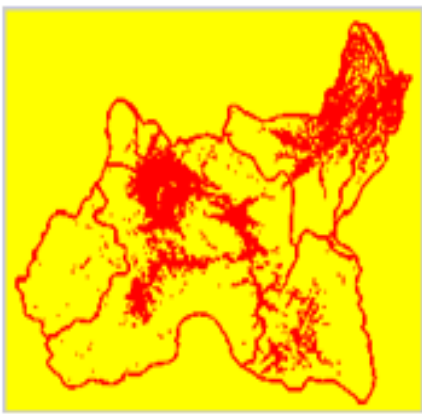

4

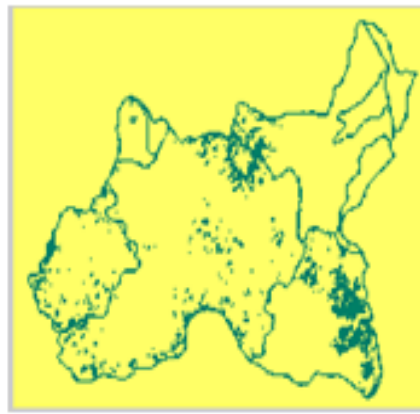

2

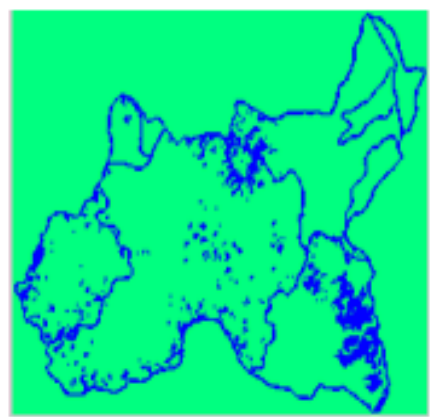

5

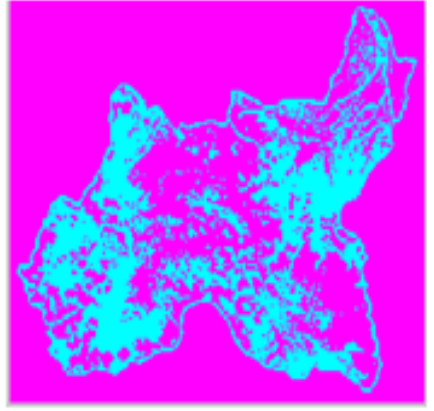

3

Legend

1 Waters

2 Woodland

3 Grass

4 Towns

5 Farmland

Figure 7. Daxiahe land use type classification map 
Table 4. NDVI values for different land use types

\begin{tabular}{c|c|c|c|c}
\hline Types & Min & Max & Stdev & Total \\
\hline Waters & -0.76625 & 0.59237 & 0.104546 & 7014712 \\
Grassland & -0.4982 & 0.62051 & 0.198214 & 10064524 \\
Town & -0.74373 & 0.600615 & 0.126054 & 8560026 \\
farmland & -0.72819 & 0.624793 & 0.130765 & 542308 \\
woodland & -0.70428 & 0.606539 & 0.362047 & 729353 \\
\hline
\end{tabular}

According to Equation 1, the different land use types of average NDVI value of the heavy metal content in the research area, precipitation, temperature factors, in order to compare the influence of different land use types on heavy metal content, the average rainfall and temperature in different areas of the process, to get the precipitation and temperature in the whole area. The corresponding NDVI value, precipitation and temperature, etc. were substituted into the corresponding formula. The results are shown in Table 5.

Table 5. Ecological risk levels of different land use types in the Daxia River Basin

\begin{tabular}{c|c|c|c}
\hline Types & HMER & Risk level & Potential ecological risk index \\
\hline Waters & -21.5568 & $\mathrm{~A}$ & Low \\
Grassland & 113.5366 & $\mathrm{~A}$ & Low \\
Town & 168.7537 & $\mathrm{~B}$ & Medium \\
Farmland & 144.7092 & $\mathrm{~A}$ & Low \\
Woodland & 69.37197 & $\mathrm{~A}$ & Low \\
\hline
\end{tabular}

It can be seen from Table 5 that the waters are negative and do not participate in the discussion. The potential risk index of grassland, farmland and forest land is low pollution, and the HMER index of woodland is the lowest. It can be inferred that the planting of woodland in this study area can help inhibit the heavy metals and reduce the ecological risk. The ecological risk level of the town is medium. Due to the large urban population, human activities are frequent. It can be seen that the impact of human activities on soil heavy metal content is positive, so the ecological risk level of towns is high.

\section{Application of different land use types in different regions}

Read, mosaic, crop, and atmospheric correction of remote sensing images with less cloud content by using ENVI software. The land is classified using unsupervised classification K-Means and combined with on-site surveys. Then, using mask technology, according to the administrative boundaries of each small area, it is divided and the NDVI value is calculated, as shown in Figure 8 and use the average of the precipitation in each area from 2015 to 2017 and the temperature average to be substituted into Equations 1 and 3, as shown in Table 6.

It can be seen from Table 6 that the ecological risk pollution levels of different land use types in small areas are low or medium, among which the ecological risk levels of grassland, farmland and forest land in Dongxiang County, Linxia City, Llinxia County, 
Hezuo City, Xiahe county, Hezheng county, Xunhua Salar Autonomous County and Tongren County are all low. The ecological risk levels of Dongxiang County, Linxia City, Linxia County, Hezuo city, Xiahe County, Hezheng County and Xunhua Salar Autonomous County were all medium, while the ecological risk levels of Tongren County were low.

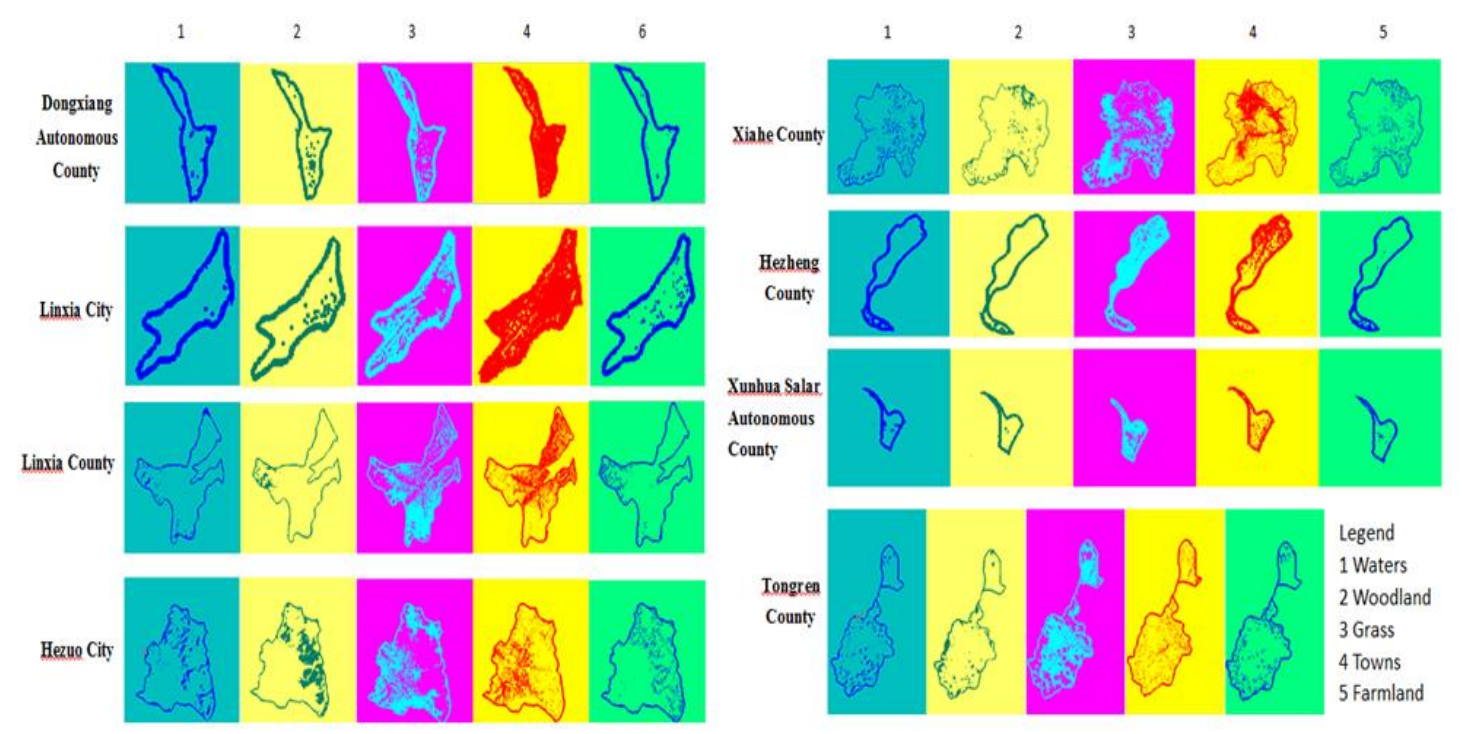

Figure 8. Small area land use type

Table 6. Ecological risk levels of different land use types in small areas

\begin{tabular}{c|c|c|c|c|c|c|c|c|c|c}
\hline \multirow{2}{*}{ Region } & \multicolumn{2}{|c|}{ Grass } & \multicolumn{2}{c|}{ Towns } & \multicolumn{2}{c|}{ Farmland } & \multicolumn{2}{c|}{ Woodland } & Waters \\
\cline { 2 - 9 } & P-I & P-L & P-I & P-L & P-I & P-L & P-I & P-L & \\
\hline Dongxiang County & 85.365 & A-L & 243.455 & B-M & 143.4 & A-L & 70.365 & A-L & No \\
Linxia City & 83.215 & A-L & 270.532 & B-M & 148.653 & A-L & 68.447 & A-L & No \\
Linxia County & 97.117 & A-L & 184.663 & B-M & 146.637 & A-L & 72.256 & A-L & No \\
Hezuo City & 132.419 & A-L & 168.321 & B-M & 84.571 & A-L & 40.752 & A-L & No \\
Xiahe County & 140.327 & A-L & 179.988 & B-M & 79.634 & A-L & 56.766 & A-L & No \\
Hezheng County & 63.021 & A-L & 193.365 & B-M & 139.521 & A-L & 86.329 & A-L & No \\
Xunhua Salar Autonomous County & 86.130 & A-L & 153.654 & B-M & 136.774 & A-L & 12.114 & A-L & No \\
Tongren County & 74.557 & A-L & 68.776 & A-L & 66.521 & A-L & 38.123 & A-L & No \\
\hline
\end{tabular}

A-L: A-low; B-M: B-medium; P-I: pollution index; P-L: pollution level

Combined with the remote sensing image map (Fig. 5) and the local interview survey, we can see that there are many towns in Dongxiang county, Linxia city and Linxia county. Human activities (factory emissions, transportation, etc.) cause high ecological risk assessment, and the ecological risk pollution index is close to 150 (close to c-heavy). Therefore, Dongxiang County, Linxia City and Linxia County can increase the planting of forest land to alleviate the ecological risk of this area. 


\section{Conclusion}

From Jan 2015 to Dec 2017, the NDVI of the study area increased, in areas where humans live in more areas, the NDVI value is lower. The NDVI is significantly affected by precipitation and temperature. The correlation coefficient between precipitation and NDVI is 0.807 , and the correlation coefficient between temperature and NDVI is 0.808 . It can be seen that the temperature has a greater influence on the NDVI. The heavy metal content was negatively correlated with temperature and precipitation, and the correlation coefficients were -0.79 and -0.784 , respectively.

The ecological risk level of land use types in grassland, cultivated land and forest land in the Daxia River Basin is low. The town is medium. The overall ecological risk is better. The ecological risk pollution levels of different land use types in small areas are also low or medium, including the grasslands of Dongxiang County, Linxia City, Linxia County, HeZuo City, Xiahe County, Hezheng County, Xunhua Salar Autonomous County and Tongren County. The ecological risk grades of cultivated land and forest land are all low. The land use types are Dongxiang County, Linxia City, Linxia County, Hezuo City, Xiahe County, Hezheng County, Xunhua Salar Autonomous County, and the ecological risk level of Tongren County is low. The towns of Dongxiang County, Linxia City and Linxia County are more distributed, and their ecological risk pollution index is close to heavy pollution. Therefore, Dongxiang County, Linxia City and Linxia County can increase the planting of forest land to alleviate the ecological risk of the area. This paper establishes remote sensing data and average heavy metal content model in the Daxia River Basin. The ecological risk assessment HMER model and method, through data verification shows that the model has potential application value and certain degree of implementation. It laid the foundation for the subsequent heavy metal pollution of the soil in the study area.

The model under study has an intentional significance for the risk assessment of the Daxia River Basin. Whether the HMER model has a more accurate assessment effect on the basin area of other vegetation and industrial types remains to be studied by the researchers. The follow-up work of this paper is mainly to study the data monitoring through data sensors and establish intelligent data detection and analysis in order to increase the efficiency of the relevant staff.

Acknowledgments. This work was supported by the National Natural Science Foundation of China under Grant No.61563047.

\section{REFERENCES}

[1] Ahonen, S. A., Hayden, B., Leppänen, J. J., Kahilainen, K. K. (2018): Climate and productivity affect total mercury concentration and bioaccumulation rate of fish along a spatial gradient of subarctic lakes. - Science of the Total Environment 637: 1586-1596.

[2] Chen, N. H. (2015): Discussion on the causes and countermeasures of water environment problems in Linxia Prefecture. - Agricultural Science \& Technology and Information, (12): 91-92.

[3] Doabi, S. A., Karami, M., Afyuni, M., Yeganeh, M. (2018): Pollution and health risk assessment of heavy metals in agricultural soil, atmospheric dust and major food crops in Kermanshah province, Iran. - Ecotoxicology and Environmental Safety 163: 153-164.

[4] Hakanson, L. (1980): An ecological risk index for aquatic pollution control. A sedimentological approach. - Water Research 14(8): 975-1001. 
[5] Li, Q., Li, T. L., Liu, D. X., Jin, Z. H. (2011): Characteristics of rainwater runoff pollution in different land use types in Tianjin. - Environmental Pollution \& Control 33(7): 22-26.

[6] Li, Y. W., Cao, C., Ju, T. Z., Zhao, X. T., Mu, R. Q., Liu, S. Q., Du, M. Z (2015): Soil heavy metal pollution characteristics and ecological risk assessment of vegetable soils in different areas of Baiyin City. - Journal of Ecology 34(11): 3205-3213.

[7] Liang, B., Nie, X. G., Yang, D. S., Wan, D., Fang, J. P., Zhao, W.(2018): Analysis of soil physical properties of five typical land use patterns in the lower reaches of the Niyang River Basin in Tibet. - Journal of Northwest A\&F University (Natural Science Edition) 46(1): 119-128.

[8] Lin, Y. H. (2015): Investigation and analysis of water environment status and pollution causes in the Daxia River Basin. - People's Yellow River 37(1): 74-78.

[9] Liu, J. L., Zhang, J. T. (2019): Current status and treatment of heavy metal pollution in soil. - Shandong Industrial Technology 7: 209.

[10] Liu, W., Zheng, B. H., Fu, Q., Luo, Y. P., Wang, M. (2013): Application of water pollution index method in river water quality evaluation. - Environmental Monitoring in China 29(3): 49-55.

[11] Liu, Y., Li, L., Chen, X., Zhang, R., Yang, J. (2018): Temporal-spatial variations and influencing factors of vegetation cover in Xinjiang from 1982 to 2013 based on GIMMSNDVI3g. - Global and Planetary Change 169: 145-155.

[12] Luo, R. Q. (2017): Discussion on the causes and countermeasures of water environment problems in Linxia Prefecture. - Agricultural Science \& Technology 2: 36-37.

[13] Sawut, R., Kasim, N., Maihemuti, B., Hu, L., Abliz, A., Abdujappar, A., Kurban, M. (2018): Pollution characteristics and health risk assessment of heavy metals in the vegetable bases of northwest China. - Science of the Total Environment 642: 864-878.

[14] Shi, R. G., Zhang, Y. W., Xu, M. M., Zheng, X. Q., Zhao, Z. S. (2019): Evaluation and source analysis of heavy metal pollution in soils in Tianjin Suburbs. - Journal of AgroEnvironment Science 38(5): 1069-1078.

[15] Su, W., Chen, M. H., Shen, G. S. (2013): Distribution characteristics and ecological risk of heavy metal $\mathrm{Pb}$ in soils of different land use types in suburbs of Changchun City. Journal of Anhui Agricultural Sciences 41(31): 12303-12305.

[16] Tang, M., Zhao, X. N., Gao, X. D., Zhang, C., Wu, P. (2018): Characteristics of soil moisture in different land use types in loess hilly region. - Chinese Journal of Applied Ecology 29(3): 765-774.

[17] Wang, S. P., Lian, B. (1993): Characteristics and distribution of soil environmental background value in Gansu Province. - Journal of Gansu Environmental Research and Monitoring 23(3): 1-7.

[18] Wang, S. Z. (2016): Discussion on planning and design of Daxiahe flood control project in Xiahe County, Gannan Prefecture. - Journal of Gansu Agriculture (20): 39-41.

[19] Wang, X., Ciais, P., Wang, Y., Zhu, D. (2018): Divergent response of seasonally dry vegetation to climatic variations in dry and wet seasons. - Glob Change Biol 24: 47094717.

[20] Wang, Z. X., Guo, Q. W., Yang, Z. H., Sun, G. Q., Ye, W. S., Hu, X. B. (2016): A land use-based spatial analysis method for human health risk assessment of heavy metals in soil and its application in Zhuzhou City, Hunan Province, China. - Journal of Central South University 23(8): 1915-1923.

[21] Wu, J. N., Long, J., Liu, L. F., Wu, Q. S., Huang, B. C., Zhang, J. M. (2018): Characteristics and evaluation of heavy metal pollution in soils of different land use types in typical lead-zinc mineralization areas. - Earth and Environment 46(6): 561-570.

[22] Yang, Q., Li, Z., Lu, X., Duan, Q., Huang, L., Bi, J. (2018): A review of soil heavy metal pollution from industrial and agricultural regions in China: pollution and risk assessment. - Science of the Total Environment 642: 690-700. 
[23] Yang, Z. H. (2013): Discussion on water quality status assessment and protection measures in Daxia River Basin. - Gansu Science and Technology 31(6): 38-40.

[24] Yu, K., Van Geel, M., Ceulemans, T., Geerts, W., Ramos, M. M., Serafim, C., ... Ameglio, T. (2018): Vegetation reflectance spectroscopy for biomonitoring of heavy metal pollution in urban soils. - Environmental Pollution 243: 1912-1922.

[25] Yuan, S. C., Gu, F. G. (2018): Study on vegetation coverage classification based on normalized index (NDVI) taking Guizhou Province as an example. - Environmental Technology 24(3): 38-42.

[26] Zhou, P., Wen, A. B., Shi, Z. L., Yan, D. C., Long, Y. (2017): Distribution characteristics and pollution evaluation of heavy metals in different land use soils in the Three Gorges Reservoir Area. - Transactions of the Chinese Society of Agricultural Machinery 48(7): 207-213.

[27] Zhou, Y., Aamir, M., Liu, K., Yang, F., Liu, W. (2018): Status of mercury accumulation in agricultural soil across China: spatial distribution, temporal trend, influencing factor and risk assessment. - Environmental Pollution 240: 116-124. 